\title{
Saúde sexual e reprodutiva da mulher com transtorno mental: fatores que moldam a assistência profissional
}

\author{
Sexual and reproductive health of women with mental disorder: factors shaping professional care \\ Salud sexual y reproductiva de las mujeres con trastorno mental: factores que determinan la \\ atención profesional
}

Recebido: 27/03/2021 | Revisado: 04/04/2021 | Aceito: 08/04/2021 | Publicado: 19/04/2021

\author{
Daniela Claudia Silva Fortes \\ ORCID: https://orcid.org/0000-0003-3539-2895 \\ Universidade Federal do Rio Grande, Brasil \\ E-mail: danielafortes93@gmail.com \\ Mara Regina Santos da Silva \\ ORCID: https://orcid.org/0000-0002-7385-7609 \\ Universidade Federal do Rio Grande, Brasil \\ E-mail: marare2021@gmail.com \\ Kateline Simone Gomes Fonseca \\ ORCID: https://orcid.org/0000-0002-2850-0352 \\ Universidade Federal do Rio Grande, Brasil \\ E-mail: kekyssskate@gmail.com \\ Ariana Sofia Barradas da Silva \\ ORCID: https://orcid.org/0000-0003-1134-1823 \\ Universidade Federal do Rio Grande, Brasil \\ E-mail: arianaso.silva@student.unicv.edu.cv
}

\begin{abstract}
Resumo
A saúde sexual e reprodutiva da mulher com transtorno mental é um tema atual e emergente. Os profissionais de saúde possuem papel importante no atendimento das necessidades de saúde dessas mulheres, no entanto nem sempre estão preparados para prestar um cuidado resolutivo. O presente estudo teve por objetivo identificar os fatores que moldam a assistência de saúde sexual e reprodutiva à mulher com transtorno mental, nos serviços de saúde em São Vicente/ Cabo Verde. Trata-se de um estudo exploratório com abordagem qualitativa, realizado com 17 profissionais que trabalham na rede de serviços de saúde da ilha de São Vicente em Cabo Verde. Os dados foram coletados por meio de entrevistas semiestruturadas, entre setembro e outubro de 2018 e submetidos à análise temática. Os resultados evidenciam que fatores culturais, educacionais e políticos são moduladores da prática profissional, no campo da saúde sexual e reprodutiva das mulheres com transtorno mental. Faz-se necessário planejar intervenções que levem em consideração a influência dos fatores culturais, educacionais e políticos na prática dos profissionais, com vistas a melhorar, a médio e longo prazo, a assistência a esse grupo de mulheres.
\end{abstract}

Palavras-chave: Cuidados de saúde; Pessoal de saúde; Mulheres; Saúde sexual; Saúde reprodutiva; Enfermagem.

\begin{abstract}
The sexual and reproductive health of women with mental disorders is a current and emerging issue. Health professionals have an important role in meeting the health needs of these women, however, they are not always prepared to provide a resolute care. This study aimed to identify the factors that shape the sexual and reproductive health care of women with mental disorders in health services in São Vicente/Cape Verde. This is an exploratory study with a qualitative approach, carried out with 17 professionals working in the health services network on the island of São Vicente in Cape Verde. Data were collected through semi-structured interviews between September and October 2018 and submitted to thematic analysis. The results show that cultural, educational and political factors are changers of professional practice in the field of sexual and reproductive health of women with mental disorder. It is necessary to plan interventions that take into account the influence of cultural, educational and political factors on the practice of professionals, in order to improve, in the medium and long term, the assistance to this group of women.
\end{abstract}

Keywords: Health care; Health personnel; Women; Sexual health; Reproductive health; Nursing.

\section{Resumen}

La salud sexual y reproductiva de las mujeres con trastornos mentales es un tema actual y emergente. Los profesionales de la salud tienen un papel importante a la hora de satisfacer las necesidades sanitarias de estas mujeres, sin embargo, no siempre están preparados para ofrecer una atención resolutiva. Este estudio tuvo como objetivo identificar los factores que conforman la atención en salud sexual y reproductiva a mujeres con trastornos mentales en 
los servicios de salud de São Vicente/Cabo Verde. Se trata de un estudio exploratorio con enfoque cualitativo, realizado con 17 profesionales que trabajan en la red de servicios sanitarios de la isla de São Vicente, en Cabo Verde. Los datos se recogieron mediante entrevistas semiestructuradas entre septiembre y octubre de 2018 y se sometieron a un análisis temático. Los resultados muestran que los factores culturales, educativos y políticos están cambiando la práctica profesional en el ámbito de la salud sexual y reproductiva de las mujeres con trastornos mentales. Es necesario planificar intervenciones que tengan en cuenta la influencia de los factores culturales, educativos y políticos en la práctica de los profesionales, con vistas a mejorar, a medio y largo plazo, la asistencia a este grupo de mujeres.

Palabras clave: Atención sanitaria; Personal sanitario; Mujeres; Salud sexual; Salud reproductiva; Enfermería.

\section{Introdução}

Um dos grandes desafios para as mulheres com transtorno mental é a dificuldade que encontram no acesso aos serviços de saúde e no cuidado a sua saúde sexual e reprodutiva. Geralmente o próprio sistema de saúde pode se configurar como barreira, mesmo quando existem diretrizes para organizar a prática dos profissionais. Inúmeros fatores se configuram como barreiras, dentre esses o fraco investimento nas estruturas sociais e institucionais através das quais os cuidados são desenvolvidos; profissionais não qualificados; desarticulação entre os serviços que constituem a rede de atendimento, entre outros (Olofsson, Sebastian \& Jegannathan, 2018; Manakampe, 2020). Destaca-se, ainda, que em contextos nos quais os recursos disponíveis e a infraestrutura de saúde estão abaixo do recomendado, tem-se observado impacto negativo na organização dos serviços e nas práticas dos profissionais que respondem que atendem essas mulheres.

Atitudes dos profissionais de saúde também influenciam nas práticas, pois podem estar baseadas em suposições equivocadas fomentando a negligência das necessidades de saúde dessa população, distanciando-as cada vez mais dos cuidados de saúde que precisam (Vistorte et al, 2018; Whylee \& Olivier, 2020). Essas atitudes são determinadas por um conjunto de fatores sociais, sistêmicos e a visão de mundo dos profissionais de saúde, que consequentemente moldam sua assistência, gerando muitas vezes uma prática negligente com essas mulheres (Puddifoof, 2017; Macdonald, FainmanAdelman, Anderson \& Iyer, 2018).

Na ilha de São Vicente/ Cabo Verde/ África os cuidados de saúde sexual e reprodutiva da mulher com transtorno mental são realizados de forma semelhante. Desde a sua independência em 1975, o país tem conseguido ganhos incontestáveis em matéria de saúde sexual e reprodutiva, tendo um arcabouço legal, recurso humano capacitado, estruturas de saúde satisfatórias, programas e políticas de saúde. Contudo, a organização e o funcionamento do sistema de saúde têm apresentado certos entraves para o Programa Nacional de Saúde Reprodutiva, com limites e desafios a serem superados (Villela, Lima \& Brito, 2018).

Nesse contexto, a prestação dos cuidados à saúde sexual e reprodutiva sofre influência de informações distorcidas e permeadas pelo juízo de valores e as características socioculturais e políticas do país, incluindo a autonomia das mulheres, entre outros (Villela et al., 2018). Ademais se constata que mesmo nos estudos que envolvem as populações em vulnerabilidade como os profissionais do sexo, homossexuais, usuários de drogas, a mulher com transtorno mental não é foco e consequentemente não é alvo de cuidado. Apesar disso, o cuidado a saúde sexual e reprodutiva das mulheres em geral, requer intervenções eficazes e diferenciados que atendam as suas necessidades, e que superam as barreiras de caráter socioculturais ou estruturais existentes. Tratando-se da mulher com transtorno mental exige um cuidado mais direcionado que leve em consideração as especificidades que a doença mental acarreta e os desafios e limitações que geralmente enfrentam para receber um cuidado resolutivo nos serviços de saúde (Hameed et al., 2020).

Este estudo tem por objetivo identificar os fatores que moldam a assistência de saúde sexual e reprodutiva à mulher com transtorno mental, nos serviços de saúde em São Vicente/ Cabo Verde. 


\section{Metodologia}

Trata-se de um estudo exploratório, de natureza qualitativa que aborda a assistência à saúde sexual e reprodutiva prestada às mulheres com transtorno mental, na ilha de São Vicente em Cabo Verde/África. A natureza qualitativa deste estudo possibilitou a apreensão e compreensão dos diferentes fatores e concepções acerca da saúde sexual e reprodutiva naquele contexto e a influência destas na assistência prestada. A pesquisa qualitativa trabalha com a natureza dos significados, dos motivos, das crenças, valores e também as atitudes, que podem ser entendidos como um conjunto de fenômenos que fazem parte da realidade social do indivíduo (Minayo, 2013; Pereira et al., 2018). Já a natureza exploratória permitiu maior familiaridade com o fenômeno estudado, tornando-o mais explícito (Gil, 2017).

O estudo foi desenvolvido com 17 profissionais de saúde que atuam em estruturas de cuidados primários e no serviço de psiquiatria, localizados na ilha de São Vicente, em Cabo Verde/África. A organização do Serviço Nacional de Saúde do setor público está organizada em três níveis: Municipal, Regional e Central que trabalham em articulação. O nível Municipal responde aos cuidados primários e constitui a porta de entrada para o Serviço Nacional de Saúde; o nível Regional reorganiza as estruturas sanitárias dos municípios mais próximos em uma ilha, com ênfase no cuidado de atenção secundária e hospitalar, e por fim o nível Central ou de referência que da cobertura a nível nacional, prestando os cuidados de atenção terciária, sobretudo, a função hospitalar diferenciada (Política Nacional de Saúde, 2007).

Para a coleta dos dados utilizou-se entrevistas semiestruturadas, realizadas por meio de videoconferência entre Brasil e Cabo Verde nos meses de setembro e outubro de 2018, tendo no local da coleta o suporte de duas auxiliares de pesquisa. Este processo iniciou após a pesquisa ser aprovada pelos Comitês de Ética e Pesquisa da Área da Saúde, da Universidade Federal do Rio Grande CEPAS/FURG, sob o parecer n 86/2018 e do Comitê Nacional de Ética em pesquisa para a Saúde (CNEPS), do Ministério de Saúde e Segurança Social de Cabo Verde, pela deliberação no 34/2018, local de procedência dos dados. Foram respeitados os preceitos da Resolução 466/12 do Conselho Nacional de Saúde, uma vez que o estudo esteve vinculado a uma instituição brasileira.

As entrevistas foram gravadas e, posteriormente, transcritas na íntegra. O material obtido foi submetido à análise de conteúdo na modalidade de temática preconizada por Bardin (2006) que envolveu uma pré-analise (correspondente a leitura flutuante do material, seguindo a definição do corpus de análise, a definição dos objetivos, o recorte do texto em unidades comparáveis de categorização para a análise temática, a preparação do material para as fases seguintes); exploração do material (foi feito a definição das categorias; identificação das unidades de registro e, sequencialmente, as unidades de significado e unidades de contexto) e, por fim, a interpretação dos resultados.

\section{Resultados}

Os resultados são apresentados em duas seções: a primeira referente a caracterização dos participantes e a segunda que aborda os fatores que influenciam a assistência a saúde sexual e reprodutiva da mulher com transtorno mental. 
Tabela 1. Caracterização dos participantes.

\begin{tabular}{|c|c|}
\hline \multirow{2}{*}{\multicolumn{2}{|c|}{$\begin{array}{l}\text { Variáveis } \\
\text { Sexo }\end{array}$}} \\
\hline & \\
\hline Masculino & 5 \\
\hline Feminino & 12 \\
\hline \multicolumn{2}{|l|}{ Profissão } \\
\hline Enfermeiros(as) & 11 \\
\hline Médicos(as) & 4 \\
\hline Psicólogos(as) & 2 \\
\hline \multicolumn{2}{|l|}{ Estado Civil } \\
\hline Solteiro & 8 \\
\hline Casado & 7 \\
\hline Divorciado & 1 \\
\hline União estável & 1 \\
\hline \multicolumn{2}{|l|}{ Orientação Religiosa } \\
\hline Católicos & 6 \\
\hline Evangélicos & 1 \\
\hline Espírita & 1 \\
\hline Ateu & 1 \\
\hline Sem informação & 8 \\
\hline \multicolumn{2}{|l|}{ Faixa etária } \\
\hline 30-39 anos & 9 \\
\hline 40-49 anos & 2 \\
\hline 50-59 anos & 3 \\
\hline 60-69 anos & 3 \\
\hline \multicolumn{2}{|l|}{ Área trabalho } \\
\hline Saúde Sexual e Reprodutiva & 12 \\
\hline Saúde Mental & 5 \\
\hline \multicolumn{2}{|l|}{ Grau escolaridade } \\
\hline Graduação/Licenciatura & 12 \\
\hline Mestrado & 2 \\
\hline Especialidade & 3 \\
\hline \multicolumn{2}{|l|}{ Tempo formação } \\
\hline $3-14$ anos & 10 \\
\hline 22-38 anos & 7 \\
\hline \multicolumn{2}{|l|}{ Tempo de vínculo } \\
\hline $1-11$ anos & 11 \\
\hline 15-30 anos & 6 \\
\hline
\end{tabular}

Fonte: Autores.

\section{Fatores que influenciam a assistência a saúde sexual e reprodutiva da mulher com transtorno mental}

\section{O fator cultural}

O fator cultural foi apontado pelos participantes como uma das principais barreiras para uma assistência resolutiva à saúde sexual e reprodutiva das mulheres com transtorno mental. Os estigmas referentes à doença mental constituem-se como fatores fortemente enraizados no contexto cabo-verdiano, que se manifestam pelo descrédito nas competências e na autonomia dessas mulheres. Embora os participantes considerem que os estigmas estão vinculados somente a família e a sociedade, quando convidados a refletir sobre a influência desta questão na prática que desenvolvem reconhecem que, por partilhar a mesma cultura, esses fatores lhes impedem de reconhecer as competências da mulher com transtorno mental para se cuidar e vivenciar sua vida sexual e reprodutiva com responsabilidade.

A nossa sociedade vê o doente mental como um incapaz, uma pessoa que não tem poder de decisão, uma pessoa sem autonomia... É um problema sério porque mudar a cultura não é fácil, trabalhar a cultura de um povo não é tão simples... Fazemos parte dessa cultura, muitas vezes, de forma inconsciente, carregamos essa mesma concepção, no dia-a-dia da prática $(P 1)$. 
Na nossa sociedade as pessoas com transtorno mental são mal vistas, quando chegam às instituições para procurar assistência de saúde sexual e reprodutiva ou por outra questão como marcar uma consulta as pessoas já olham para elas de forma errada (P16).

O discurso dos participantes evidenciou uma ambigüidade. Por um lado reconhecem que qualquer pessoa deve gozar plenamente seus direitos sexuais e reprodutivos, e vivenciar de forma livre e segura sua vida sexual e reprodutiva. No entanto, quando questionados sobre as ações desenvolvidas com as mulheres com transtorno mental a resposta indicava um cuidado com foco no controle da natalidade e justificaram suas ações com base nas capacidades dessas mulheres prejudicadas pelo transtorno mental.

Há casos em que se propõem métodos de longa duração como os implantes, ou a laqueação das trompas porque sabemos que outra coisa não seria possível, para evitar uma gravidez... Há situações que a própria família pede para se fazer a esterilização e há outros casos em que alguns técnicos decidem ou digamos uma conferência entre as várias instituições e vê-se o que seria melhor para essa mulher (P14).

A visão de mundo que alguns profissionais partilhavam em relação à mulher com transtorno mental ficou manifesto no seu discurso em palavras tais como "incapacidade"; "exacerbado"; "manifestação da doença mental"; "não tem condições para cuidar de um filho"; "ela é dependente"; "geralmente está descompensada"; "ela é vulnerável”; "podem abusar sexualmente dela". Essa visão de mundo pode estar associada aos fatores culturais que presumem que a mulher responda a determinados papeis como a de boa mãe e boa esposa, de certo modo, a mulher com transtorno mental ficou patente no discurso desses profissionais como alguém que não enquadra para responder positivamente a esses papeis.

\section{O fator educacional}

De forma unânime, os participantes referem que não se sentem preparados para lidar com as questões complexas que envolvem a saúde sexual e reprodutiva da mulher com transtorno mental. O despreparo não se refere às competências técnicocientíficas necessárias a prestação da assistência às mulheres em geral, mas, as dificuldades são especificas quando se trata de mulheres com um transtorno mental.

A abordagem resolutiva comprometida à saúde desse grupo de mulheres foi mencionada pelos participantes como consequência de um despreparo que remonta desde o percurso acadêmico reforçando uma lacuna em relação ao conteúdo de saúde sexual e reprodutiva da mulher com transtorno mental no processo de formação. Na percepção dos participantes isso contribuiu para uma maior dificuldade em reconhecer e responder as necessidades de saúde sexual e reprodutiva desse grupo de mulheres.

Não tive experiências, nem orientações de como proceder em relação às questões de saúde sexual e reprodutiva, nesses casos, o que estudamos era saúde mental e como lidar com o paciente internado (P10).

Posso afirmar que não recebi nenhuma orientação em relação à saúde sexual e reprodutiva dos doentes mentais, aprendi sim de forma geral sobre a saúde sexual e reprodutiva (P3).

Os profissionais referiram ainda, que mesmo na formação continuada no serviço, esse é um tema que geralmente passa despercebida. Frisaram, também, que constantemente recebem formações direcionadas à saúde sexual e reprodutiva, mas nada em relação à saúde da mulher com transtorno mental o que, no seu entendimento, influencia na prática assistencial. Nesse contexto, constata-se que a saúde sexual e reprodutiva da mulher com transtorno mental é um tema carente de atenção desde as políticas de saúde à prática assistencial. 
Nós recebemos muitas formações, mas, no entanto, muito pouco se fala da atenção à saúde sexual e reprodutiva da mulher com transtorno mental (P7).

Nós profissionais estamos pouco preparados e por vezes nem sabemos como lidar e evitamos tocar no assunto e nem deixamos a mulher falar muito do assunto, desde que trabalho aqui estou sempre lutando, reivindicando formação continuada no serviço, e em relação a esse assunto em concreto precisamos muito que nos capacitem (P5).

Alguns participantes reconhecem que a mulher com transtorno mental é duplamente prejudicada. De um lado apontaram as fragilidades no preparo dos profissionais de saúde e de outro a família que com fraco conhecimento também apresentam limitações em identificar e compreender as necessidades de saúde sexual e reprodutiva da mulher com transtorno mental. Os participantes reconheceram que precisam estar capacitados para poder trabalhar as famílias e ofertar um melhor cuidado as mulheres.

Eu penso que as famílias também não estão preparadas e este seria o nosso papel: ajudá-las a entender as necessidades que as pessoas têm de se relacionar sexualmente, mesmo tendo um transtorno mental (P7).

Para esses participantes a educação continuada configura o melhor investimento na capacitação dos recursos humanos, apontando que ações que trabalhem também a visão de mundo podem apresentar potencialidades úteis para um melhoramento na resposta dispensado a saúde sexual e reprodutiva da mulher com transtorno mental nos serviços de saúde envolvidos neste estudo.

\section{O fator político}

Os participantes referem que os programas e políticas de saúde do Ministério da Saúde e Segurança Social de Cabo Verde são amplos e generalizados. Salientam a necessidade de um trabalho mais inclusivo que reconheça as mulheres com transtorno mental, firmando ações que promovam a equidade na procura e oferta dos cuidados a sua saúde. Além disso, que a organização dos serviços de saúde foi apontada como responsável pelo não reconhecimento da mulher com transtorno mental nesses serviços de saúde.

Nós seguimos os programas do Ministério de Saúde, mas são generalizados não havendo algo que te norteie em específico a mulher com transtorno mental (P12).

Nós seguimos os programas e as políticas, quer dizer nós tentamos porque às vezes o que vemos é que os programas e políticas estão perfeitos no papel, mas no terreno deparamos com muitas dificuldades principalmente no que tange aos recursos humanos capacitados para dar respostas a essas políticas (P7).

Os profissionais foram unânimes que a organização da assistência precisa de uma abordagem mais horizontal que resolva o problema desde as políticas e programas existentes a formulação de diretrizes e protocolos de serviço. A inexistência de protocolos de serviço foi elucidada como uma falha que prejudica a assistência à saúde sexual e reprodutiva da mulher com transtornos mentais, uma vez que, não possuam um documento que sirva de guia para orientá-los. Os participantes reconheceram que nem sempre estão preparados para responder as necessidades de saúde sexual e reprodutiva deste grupo de mulheres, e consideraram que os protocolos de serviço poderiam efetivar-se como meio facilitador e orientador da sua prática assistencial.

Aqui na enfermaria nós não temos nenhum protocolo que poderíamos seguir que nos diga como fazer em caso dessas situações (P4). 
Não vi nenhum protocolo, o que orientava os colegas é a experiência que tinha e alguns estudos. Era isso, conhecimento e experiência adquirida, porque protocolo que eu saiba não tem (P6).

Um participante referiu que o fraco investimento em políticas públicas voltadas à saúde mental, por parte do Serviço Nacional de Saúde, contribui para que esta população tenha dificuldades em ser incluída nos cuidados de saúde. No seu entendimento, isso é um aspecto que influencia diretamente na organização e oferta dos cuidados dispensados à saúde sexual e reprodutiva dessas mulheres.

Aqui no hospital carecemos muito de protocolos entre as enfermarias e é claro que influencia. O paciente é visto como um doente mental, eles não vêem o paciente holisticamente como uma pessoa como um todo, é visto uma parte (P5).

Em síntese, os participantes reconhecem a necessidade de maior visibilidade da mulher com transtorno mental nos programas e políticas existentes no país sobre a saúde sexual e reprodutiva. Ao mesmo tempo, sentem a necessidade de maior capacitação e documentos orientadores para que possam ter maior aptidão de respostas às necessidades desta população específica.

\section{Discussão}

Os resultados sugerem que os fatores culturais, educacionais e políticos são vistos pelos participantes como fatores que moldam a sua assistência influenciando no cuidado dispensado a saúde sexual e reprodutiva da mulher com transtorno mental. Essa visão é consistente com os resultados de estudos anteriores explorados na literatura internacional chamando a atenção para uma intervenção coletiva que capacite os profissionais de saúde possibilitando que reconheçam os direitos sexuais e reprodutivos dessas mulheres. Acrescenta-se ainda a necessidade de ações que salvaguarde seus direitos e proporciona a oferta de um cuidado que responda as suas necessidades de forma integral. As intervenções realizadas com os profissionais de saúde poderão impactar diretamente na qualidade de vida, saúde sexual e reprodutiva e cuidados recebidos pelas mulheres com transtorno mental.

Os achados deste estudo deixam evidenciar que os fatores culturais de uma sociedade apresentam grande potencial para moldar a assistência dos profissionais de saúde, uma vez que, a identidade cultural faz parte do macrossistema do indivíduo. Por conseguinte, identificou-se que embora os profissionais possam estar capacitados com conhecimento técnicocientífico não é um processo simples fazer a dissociação entre a prática profissional e sua identidade cultural. Um estudo desenvolvido no Brasil sugere que as interferências culturais que agregam a identidade profissional, podem influenciar no diagnóstico e impactar na falta de acesso à informação de boa qualidade às mulheres assistidas (Soares et al., 2020).

Reconhecesse que as atitudes dos profissionais de saúde muitas vezes estão associadas ao contexto, assim sendo, usualmente suas atitudes são gerados e mantidos de maneira coletiva ou culturalmente, fazendo parte da identidade individual das pessoas. Tais atitudes moldam o comportamento socialmente enraizado e frequentemente são considerados como características de organizações e instituições, comunidades e sociedades, sendo relativamente estáveis e resistentes à mudança (Macdonald et al., 2018; Ganle, Apolot, Rugoho \& Sumankuuro, 2020; Whylee \& Olivier, 2020).

Um estudo desenvolvido em Israel demonstrou que nessas condições os fatores culturais podem apresentar-se como uma das principais barreiras na assistência influenciando adversamente a prestação de cuidados, salientando que, o ponto de vista e atitudes dos profissionais de saúde pode levar a consequências mais sérias como a perda de confiança e uma baixa procura pelos serviços de saúde sexual e reprodutiva (Daeem et al., 2019). Neste sentido, um estudo desenvolvido com países da África Subsaariana realçou a importância de investir em ações que trabalhem a visão de mundo e a identidade cultural dos 
profissionais de saúde com aposta na desconstrução de estigmas que influenciam na organização e oferta dos cuidados (Akiba et al., 2019).

O contexto cultural mostrou-se neste estudo como fator que excede a assistência dos profissionais, tendo sido apontado como influenciador de outras entidades nomeadamente os formuladores de políticas públicas, a família, a sociedade e a própria mulher. Essa mesma concepção foi apontada em um estudo que mostrou que o estigma como determinante sociocultural compartilhado por um coletivo pode ser um fator decisivo que leva às desigualdades e padronização da saúde das pessoas com transtorno mental, restringindo as suas necessidades de foro psiquiátrico (Niedzwiedz, 2019).

Aliado a intervenções que vise trabalhar com os fatores culturais dos profissionais de saúde, a capacitação dos mesmos é uma necessidade emergente para garantir os cuidados efetivos às mulheres com transtorno mental (Akiba et al., 2019). Tal fato equiparasse aos resultados do presente estudo, em que os participantes relataram não estar preparados para atender a saúde sexual e reprodutiva da mulher com transtorno mental.

O despreparo em saber lidar, reconhecer e responder as necessidades de saúde desse grupo de mulheres mostrou-se como uma barreira passível de mudança com investimento na capacitação continuada dos profissionais de saúde, e uma maior sensibilidade por parte das entidades competentes em reconhecer que o despreparo desses profissionais agrava a situação de vulnerabilidade a que esse grupo de mulheres possa estar exposto. A educação continuada nos serviços de saúde pode trazer benefícios, como melhoria na acessibilidade, aumentar a flexibilidade e fornecer uma resposta rápida às necessidades apresentadas pelo público cuidado (Rye, Fribourg \& Skre, 2019; Hadadgar et al., 2016). Embora, ao longo da formação acadêmica sejam adicionados conteúdos curriculares como, por exemplo, a medicina baseada em evidências, muitos desses acréscimos são feitos de forma fragmentada e não acompanham a evolução das necessidades da população (Petkari, Masedo, Xavier \& Moreno, 2019).

Assim, ao terminar sua formação, o profissional não tem suas competências plenamente desenvolvidas, o que o limita para a resolubilidade dos cuidados. Razão pela qual, a formação continuada nos serviços é um recurso para superar essa deficiência na capacitação dos profissionais (Laine et al., 2019).

Neste sentido, a educação continuada se afigura como um modelo inovador que trabalha com foco a transformar a realidade, contribuindo na busca do ensino-aprendizagem amplo, partindo do cotidiano do profissional de saúde e dar continuidade ao processo ensino-serviço (Godsey, Houghton, Hayes, 2020). Os resultados deste estudo se assemelham aos achados supracitados, uma vez que, o percurso acadêmico dos profissionais de saúde é parte da sua formação profissional, desse modo, a educação continuada em serviços proporcionaria a capacitação e continuidade na formação desses profissionais.

Nesse estudo, o distanciamento existente entre a saúde mental, a saúde sexual e a saúde reprodutiva, em particular, nos doentes mentais, desde o percurso acadêmico até a formação continuada nos serviços de saúde, foi alvo de crítica por parte dos participantes, como elementos que são dissociados. A educação continuada com maior articulação entre esses eixos pode efetivar-se como forma de capacitar os profissionais de saúde a dar melhor resposta a saúde sexual e reprodutiva da mulher com transtorno mental. Corroborando, um estudo desenvolvido com estudantes chama a atenção para a necessidade de maior investimento e preparação dos fornecedores de educação, incluindo as universidades e as instituições de saúde (Heim et al., 2019).

O reconhecimento das necessidades educacionais dos profissionais de saúde e um investimento em educação continuada têm potencial para o retorno, em longo prazo, em termos de melhorias tanto para o serviço de saúde, quanto para os profissionais e população assistida. Da mesma forma, o não investimento, ou o investimento precário pode contribuir para que os profissionais não melhorem sua formação profissional e pode ter agravos como manter o déficit na prática assistencial por muito tempo (Miller, Scherpbier, Amsterdam, Guedes \& Pype, 2019). 
A organização Mundial da saúde (OMS), elaborou o the mental Health Gape Action Program (nhGAP), para sua implementação em países de baixa e média renda, chamando atenção para sua implementação na formação dos profissionais de saúde desde a academia até a atualização de forma sistematizada nos serviços de saúde (Faregh, Lencucha, Ventevogel, Dubale \& Kirmayer, 2019).

Outros recursos passíveis de investimento nesse contexto são os protocolos de serviço e diretrizes clínicas para fomentar a assistência a saúde sexual e reprodutiva da mulher com transtorno mental, uma vez que, nesse estudo foram apontados como uma das barreiras que condicionam a prestação de cuidado. Os protocolos de serviço de saúde ajudam para melhor sistematização dos cuidados, organizando o processo de trabalho, bem como podem servir como orientação e suporte aos profissionais no desenvolvimento dos cuidados (Hlongwa \& Sibiya, 2019).

A inexistência de protocolos de serviços que oriente para o cuidado a essas mulheres pode influenciar na qualidade do atendimento dispensado, comprometendo um cuidado que responda as suas necessidades. Sugere-se, que isso acaba reforçando o distanciamento existente entre esses dois eixos (saúde mental e saúde sexual e reprodutiva), dificultando ainda mais a possibilidade dessas mulheres e suas famílias procurarem por esses serviços, uma vez que não consideram a assistência como resolutiva e nem esses serviços como recurso.

Recursos preventivos orientadores e capacitadores são necessários para a melhoria da assistência de saúde a população assistida, principalmente quando estes fazem parte dos grupos mais vulneráveis e passíveis de ter os seus direitos comprometidos ou negados. Um estudo desenvolvido em países de renda baixa e média (LMICs) evidenciou que uma baixa prioridade ou comprometimento insuficiente no nível governamental, para investir em políticas públicas que superem as principais barreiras para o desenvolvimento de serviços de saúde mental e ofertar um cuidado resolutivo a esses utentes, pode ter consequência não intencional em alienar essas mulheres as invés de as empoderar (Akiba et al., 2019).

Dadas as particularidades envolvidas na assistência à saúde sexual e reprodutiva da mulher com transtorno mental, compreende-se o impacto que a ausência de documentos pode efetivar na prestação do cuidado, acrescentando o fato quando os profissionais de saúde não se sentem preparados para atender as demandas de saúde a esse grupo de mulheres, o que revela uma necessidade urgente de intervenção pelas entidades responsáveis. Particularmente no contexto de Cabo Verde urge uma necessidade de maior investimento nas políticas públicas e diretrizes institucionais, bem como uma melhoria na postura dos profissionais de saúde com vista à superação das barreiras existentes (Villela et al., 2018).

Além de protocolos e políticas públicas, uma maior aposta na formação em serviço como meio de capacitar os profissionais na sua prática clínica, reforçado com evidência atualizada na área pode efetivar-se como meio para ajudar a solucionar os problemas principalmente em contexto no qual os profissionais deparam com dificuldades na resposta a saúde sexual e reprodutiva de mulheres com transtorno mental. O envolvimento da equipa multidisciplinar no seguimento de protocolos, a capacitação recorrente dos profissionais de saúde, e inclusão ativa da comunidade devem ser ponderados para uma melhor integração das mulheres com transtorno mental nos serviços de saúde sexual e reprodutiva como co-participantes e assegurar a assistência a saúde das mesmas (Hlongwa \& Sibiya, 2019).

\section{Considerações Finais}

Diante do exposto, pode-se elucidar que o estudo aponta alguns fatores considerados como moduladores na assistência a saúde sexual e reprodutiva da mulher com transtorno mental. Os fatores culturais, educacionais e políticos foram apontados como barreiras e desafios primordiais a serem melhorados e superados para que esse grupo de mulheres receba um cuidado resolutivo e efetivo as suas necessidades. O conjunto desses fatores mostrou-se interligados e influenciáveis, sugerindo que a solução para esses problemas complexos requer intervenção conjunta que responda aos fatores culturais, educacionais e políticos de forma interligada e não com ações que trabalhe de forma isolada. 
Embora os participantes elucidassem a necessidade de investimentos do macro para o micro nomeadamente a revisão das políticas existentes, ficou evidente a necessidade de uma ampliação da consciência e sensibilidade do profissional das necessidades de saúde sexual e reprodutiva da mulher com transtorno mental. No estudo ficou sugestivo que o diagnóstico de transtorno mental apresentado por essas mulheres era o principal foco influenciado pelos fatores culturais, educacionais e políticos.

Sugestões de soluções estiveram presentes nas falas dos participantes e nos artigos analisados nomeadamente investimento na capacitação anti-estigmas, formação continuada nos serviços, desmistificação das necessidades de saúde sexual e reprodutiva da pessoa com transtorno mental, desconstrução das atitudes discriminatórias em relação ao doente mental, maior horizontalidade entre os eixos saúde sexual e reprodutiva e saúde mental, revisão das políticas públicas existentes, protocolos de serviço e promoção da igualdade e equidade na saúde desses grupos.

Convém ressaltar que se apresenta consciência que melhorar ou solucionar esses problemas requer um investimento a médio longo prazo, e que por envolver questões culturais torna o processo mais delicado. Contudo, essas ações são necessárias, uma vez que, os resultados apresentados apontam para um comprometimento dos direitos sexuais e reprodutivos dessas mulheres, advindos da assistência recebida.

Cabo Verde é um país insular, assim sendo acredita-se que os resultados deste estudo não podem ser utilizados a nível nacional para efeitos de comparação, sendo que, sugerem-se estudos futuros que englobem o território nacional. Acrescenta-se ainda a necessidade de estudos que avaliem a saúde sexual e reprodutiva da mulher com transtorno mental, sob o ponto de vista dessas mulheres e suas famílias.

\section{Agradecimentos}

Agradecemos as instituições envolvidas, aos participantes e colaboradores pela disponibilidade e contribuições essenciais para o desenvolvimento deste estudo.

\section{Referências}

Akiba, C. F., Go, V., Mwapasa, V., Hosseinipour, M., Gaynes, B. N., Amberbir, A., Udedi, M., \& Pense, B. W. (2019). The Sub-Saharan Africa Regional Partnership (SHARP) for Mental Health Capacity Building: a program protocol for building implementation science and mental health research and policymaking capacity in Malawi and Tanzania. International Journal of Mental Health Systems volume, 13:70. https://doi.org/10.1186/s13033-019-0327-2

Bardin, L. (2015). Análise de Conteúdo. Edições 70.

Daeem, R., Mansbach-Kleinfeld, I., Farbstein, I., Apter, A., Elias, R., Ifrah, A., Chodick, G., \& Fennig, S. (2019). Barriers to help-seeking in Israeli Arab minority adolescents with mental health problems: results from the Galilee study. Israel Journal of Health Policy Research, 8:45. https://doi.org/10.1186/s13584-019-0315-7

Faregh, N., Lencucha, R., Ventevogel, P., Dubale, B. W., \& Kirmayer, L. J. (2019). Considering culture, context and community in mhGAP implementation and training: challenges and recommendations from the feld.International Journal of Mental Health Systems, 13:58. https://doi.org/10.1186/s13033-019-03129

Gante, J. K., Apolot, R. R., Rugoho, T., \& Sumankuuro, J. (2020). 'They are my future': childbearing desires and motivations among women with disabilities in Ghana - implications for reproductive healthcare. Reproductive Health, 17:151. https://doi.org/10.1186/s12978-020-01000-y

Gil, A. C. (2017). Como elaborar projeto de pesquisa. (6a ed.), Atlas.129p.

Godsey, J. A., Houghton, D. M., \& Hayes, T. (2020). Registered nurse perceptions of factors contributing to the inconsistent brand image of the nursing profession. Nursing Outlook, 68, 808-821. https://doi.org/10.1016/j.outlook.2020.06.005

Hadadgar, A., Changiz, T., Massielo, I., Dehghani, Z., Mirshahzadeh, N., \& Zary, N. (2016). Applicability of the theory of planned behavior in explaining the general practitioners eLearning use in continuing medical education. BMC Medical Education, 16:215. https://doi.org/10.1186/s12909-016-0738-6

Hameed, S. Maddams, A., Lowe, H., Davies, L., Khosla, R., \& Shakespeare, T. (2020). From words to actions: systematic review of interventions to promote sexual and reproductive health of persons with disabilities in low- and middle-income countries. BMJ Global Health, 5(10): e002903. https://www.ncbi.nlm.nih.gov/pmc/articles/PMC7566436/pdf/bmjgh-2020-002903.pdf

Heim, E., Henderson, C., Kohrt, B., Koschorke, M., Milenova, M., \& Thornicroft, G. (2019). Reducing mental health-related stigma among medical and nursing students in low- and middle-income countries: a systematic review. Epidemiol Psychiatr Sci, 29: e28. https://doi.org/10.1017/S2045796019000167 
Hlongwa, E.N., \& Sibiya, M. N. (2019). A practice framework to enhance the implementation of the Policy on Integration of Mental Health Care into primary health care in KwaZulu-Natal province.African Journal of Primary Health Care \& Family Medicine, 11(1), a1865. https://www.ncbi.nlm.nih.gov/pmc/articles/PMC6489161/pdf/PHCFM-11-1865.pdf

Laine, A.Valimaki, M., Loyttyniemi, E. Pekurinen, V., Marttunen, M., \& Anttila, M. (2019). The impact of a Web-Based course Concerning Patient Education for Mental Health Care Professionals: Quasi-Experimental Study. Journal of Medical Internet Research, 21 (3): e11198. 10.2196 / 11198

Macdonald, K., Fainman-Adelman,N., Anderson, K. K., \& Iyer, S. N.(2018) Pathways to mental health services for young people: a systematic review.Social Psychiatry and Psychiatric Epidemiology,53 (10): 1005-1038.https://doi.org/10.1007/s00127-018-1578-y

Munakampe, M.N. (2020). Strengthening mental health systems in Zambia. International Journal of Mental Health Systems, 14:28. https://doi.org/10.1186/s13033-020-00360-z

Miller, R. Scherpbier, N., Amsterdam, L.V., Guedes, V., \& Pype, P. (2019). Inter-professional education and primary care: EFPC position paper. Primary Health Care Research \& Development, 20(e138): 1-10.doi: 10.1017/S1463423619000653

Minayo, M. C. S. (2013). Pesquisa Social, Teoria, método e criatividade. (28a ed.), Vozes, 108p.

Niedzwiedz, C. L. (2019). How does mental health stigma get under the skin? Cross-sectional analysis using the Health Survey for England. SSM - Population Health, 8, 100433. https://doi.org/10.1016/j.ssmph.2019.100433

Olofsson, S., Sebastian, M. S., \& Jegannathan, B. (2018).Mental health in primary health care in a rural district of Cambodia: a situational analysis. International Journal of Mental Health Systems volume, 12:7. https://doi.org/10.1186/s13033-018-0185-3

Petkari, E., Masedo, G. A., Xavier, M., \& Moreno, K. B. (2018). The influence of clerkship on students' towards mental illness: a meta-analysis. Medical Education, 52 (7): 694-704. https://doi.org/10.1111/medu.13548

Cabo Verde (2007/2020). Ministério da Saúde. Política Nacional de Saúde. Reformar Para Uma Saúde Melhor. Praia. http://www.minsaude.gov.cv/

Pereira, A. S., Sthitsuka, D. M., Pereira, F. J., \&Shitsuka, R. (2018). Metodologia de Pesquisa Científica, Universidade Federal de Santa Maria. Nucleo de Tecnologia Educaçã. https://repositorio.ufsm.br/bitstream/handle/1/15824/Lic_Computacao_Metodologia-Pesquisa-Cientifica.pdf?sequence=1.

Puddifoof, K. (2019). Stereotyping Patients. Journal of social philosophy, 50 (1):69-90. https://www.ncbi.nlm.nih.gov/pmc/a rticles/PMC6559163/pdf/JOSP50-69.pdf

Rye, M., Fribourg, O., \& Skre, I. (2019). Attitudes of mental health providers towards adoption of evidence-based interventions: relationship to workplace, staff roles and social and psychological factors at work. BMC Health Services Research, 19:110. https://doi.org/10.1186/s12913-019-3933-4

Soares, J, L., Silva, I. G. B., Moreira, M. G. R., Martins, A. K. M., Rebouças, V. C. F., \& Cavalcante, E. G. R. (2020). Transcultural theory in nursing care of women with infections. Revista Brasileira de Enfermagem, 73:e20190586. http://dx.doi.org/10.1590/0034-7167-2019-0586

Villela, W. V., Lima, R. W., \& Brito, C. F. (2018). The National Reproductive Health Programme in Cape Verde: scope, limits and challenges. Ciência \& Saúde coletiva, 23(11): 3631-3636. DOI: 10.1590/1413-812320182311.32852016

Vistorte, A. O. R., Ribeiro, W., Ziebold, C., Asevedo, E., Evans-Lacko, S., Keeley, J. W., Gonçalves, D. A., Palacios, N. G., \& Mari, J. J. Clinical decisions and stigmatizing attitudes towards mental health problems in primary care physicians from Latin American countries. Plos One, 15. https://doi.org/10.1371/journal.pone.0206440

Whyle, E., \& Oliver, J. (2020). Social values and health systems in health policy and systems research: a mixed-method systematic review and evidence map. Health Policy and Planning, 35, 2020, 735-751. https://www.ncbi.nlm.nih.gov/pmc/articles/PMC7294246/pdf/czaa038.pdf 